\title{
Desempeño productivo de novillos pastoreando sudangras o sorgo forrajero nervadura marrón (BMR) durante el verano
}

\author{
Rovira, P.; Echeverría, J. \\ Instituto Nacional de Investigación Agropecuaria (INIA), Ruta 8 km 281, CP 33000, Treinta y Tres, Uruguay. \\ Tel (598) 44521047, Fax (598)445 25701. E-mail: provira@tyt.inia.org.uy.
}

\begin{abstract}
Resumen
Rovira, P.; Echeverría, J.: Desempeño productivo de novillos pastoreando sudangras o sorgo forrajero nervadura marrón (BMR) durante el verano. Rev. vet. 24: 2, 91-96, 2013. El pastoreo de verdeos de verano permite mejorar la ganancia de peso de bovinos y aumentar la capacidad de carga del predio. El objetivo del experimento fue caracterizar el forraje ofrecido por dos materiales forrajeros y evaluar el resultado en el desempeño productivo de novillos. Cincuenta y cuatro novillos ( $304 \pm 44 \mathrm{~kg}$ ) fueron asignados al azar en 2 tratamientos con 2 repeticiones: 1) sudangras (Sorghum sudanense) cv. Estanzuela Comiray y 2) sorgo forrajero (Sorghum vulgare) cv. AGT 21 nervadura marrón (brown midrib, BMR). La dotación fue de 6 novillos/ha en pastoreo rotativo de 4 parcelas. No hubo diferencias significativas $(\mathrm{p}>0,05)$ en la altura $(93 \pm 18 \mathrm{~cm})$ y disponibilidad de forraje de hojas $(2342 \pm 1208 \mathrm{~kg}$ MS/ ha), tallos (4714 $\pm 2004 \mathrm{~kg} \mathrm{MS} / \mathrm{ha})$ y forraje total $(7078 \pm 2410 \mathrm{~kg} \mathrm{MS} / \mathrm{ha}$ ) durante los 3 ciclos de pastoreo (73 días). En ambos materiales el aporte de la fracción hoja al forraje total disminuyó de un $50 \%$ (primer pastoreo) a un $25 \%$ (tercer pastoreo). La fracción hoja del sorgo BMR presentó menos porcentaje de materia seca $(\mathrm{p}<0,05)$ que el sudangras $(22,2$ y $27,4 \%$ respectivamente) y tendió $(\mathrm{p}<0,10)$ a registrar mayor digestibilidad $(67,7$ y $64,5 \%$ respectivamente). La fracción tallo del sorgo BMR fue más digestible (70,7\%) y con menor contenido de lignina $(8,1 \%)$ comparado con el sudangras $(61,5$ y 9,3\% respectivamente) $(\mathrm{p}<0,05)$. Los novillos pastoreando sorgo BMR registraron una ganancia de peso superior $(p<0,05)$ que aquellos en el sudangras $(0,945$ y $0,823 \mathrm{~kg} / \mathrm{a} / \mathrm{d}$ respectivamente) aunque no hubo diferencias significativas en el peso vivo final (388 y $378 \mathrm{~kg}$ respectivamente). La mayor calidad del forraje ofrecido por el sorgo BMR, fundamentalmente observada en la fracción tallo (principal componente durante el segundo y tercer pastoreo), determinó una mejora en el desempeño productivo de novillos durante el verano comparado con materiales forrajeros más tradicionales (sudangras).
\end{abstract}

Palabras clave: novillo, verano, sorgo, BMR.

\begin{abstract}
Rovira, P.; Echeverría, J.: Performance of steers grazing sudangrass (Sorghum sudanense) or brown midrib (BMR) sorghum (Sorghum vulgare) during summer. Rev. vet. 24: 2, 91-96, 2013. The use of annual forage crops increases average daily gain of animals and the carrying capacity of the production system during summer. The objectives of this experiment were to characterize the forage produced by two different materials of Sorghum spp. and to evaluate the response in animal production. Fifty four yearling steers $(304 \pm 44$ $\mathrm{kg}$ ) were randomly assigned to two treatments with two repetitions: 1) sudangrass (Sorghum sudanense) cv. Estanzuela Comiray, 2) brown midrib (BMR) sorghum (Sorghum vulgare) cv. AGT 21. In both cases stocking rate was 6 steers/ha in a rotational grazing system in 4 paddocks. There were no statistical differences $(\mathrm{p}>0.05)$ in crop height $(93 \pm 18 \mathrm{~cm})$ and forage availability of leaves $(2342 \pm 1208 \mathrm{~kg} \mathrm{DM} / \mathrm{ha})$, stems ( $4714 \pm 2004 \mathrm{~kg} \mathrm{DM} / \mathrm{ha})$ and total herbage mass $(7078 \pm 2410 \mathrm{~kg} \mathrm{DM} / \mathrm{ha})$ during three grazing cycles. The contribution of leaves to total forage production decreased from $50 \%$ (first grazing period) to $25 \%$ (third grazing period) for both materials. The leaves of sorghum BMR had lower dry matter $(\mathrm{p}<0.05)$ than those from sudangrass $(22.2$ y $27.4 \%$ respectively) and tended $(\mathrm{p}<0.10)$ to register higher digestibility ( 67.7 y $64.5 \%$ respectively). The stem fraction of sorghum BMR had more digestibility (70.7\%) and less lignin (8.1\%) compared with stems of sudangrass (61.5 y 9.3\% respectively) $(\mathrm{p}<0.05)$. As a result steers grazing sorghum BMR showed a greater $(\mathrm{p}<0.05)$ average daily gain than those grazing sudangrass $(0.945$ y $0.823 \mathrm{~kg} / \mathrm{a} / \mathrm{d}$ respectively), although final liveweight was not significantly affected ( 388 y $378 \mathrm{~kg}$ respectively) $(\mathrm{p}>0.05)$. The improved for-
\end{abstract}

Recibido: 2 agosto 2013 / Aceptado: 10 septiembre 2013 
age quality of sorghum BMR, mainly observed in the stem fraction (main plant component during the second and third grazing periods), determined an increase in average daily gain of steers during summer compared to traditional forage materials (sudangrass).

Key words: steer, summer, sorghum, BMR.

\section{INTRODUCCIÓN}

El uso de distintas variedades de sudangras (Sorghum sudanense) y sorgo forrajero (Sorghum vulgare) para pastoreo directo de los animales se ha generalizado en regiones ganaderas del Uruguay durante el verano. Estos materiales se destacan por tener una elevada producción de forraje por superficie $(8.000 \mathrm{a}$ $15.000 \mathrm{~kg} \mathrm{MS} / \mathrm{ha}$ ) que permite sostener una alta carga animal (4 a 6 animales/ha) durante los meses del verano. Sin embargo, las ganancias diarias de peso que se pueden alcanzar son moderadas debido a desbalances nutricionales ${ }^{10}$. Para superar parcialmente dicha limitante, recientemente se ha expandido el uso de variedades de sorgo forrajero con el gen de nervadura marrón (Brown Middle Rib o BMR; por su sigla en inglés) que le confiere a la planta menor contenido de lignina y por lo tanto mayor digestibilidad $1,12,16,17,25$.

El incremento de productividad animal debido al gen BMR ha sido ampliamente reportado para vacas lecheras utilizando ensilajes de maíz y sorgo ${ }^{11,18,23,24,}$ ${ }^{26}$. Sin embargo, la información comparando materiales de sorgo con o sin la inclusión del gen BMR en la respuesta animal de bovinos para carne utilizando forraje fresco mediante pastoreo directo es prácticamente inexistente. Se ha reportado que la utilización de sorgos BMR como forraje fresco permite superar los 0,700 $\mathrm{kg} / \mathrm{a} / \mathrm{d}$ de ganancia diaria en novillos de $300-400 \mathrm{~kg}$ de peso vivo ${ }^{10}$.

El objetivo del presente experimento fue evaluar el desempeño productivo de novillos pastoreando un sorgo forrajero BMR como alternativa al uso de sudangras, principal opción de verdeo de verano para los sistemas ganaderos en Uruguay.

\section{MATERIAL Y MÉTODOS}

El experimento se desarrolló entre el 18 de diciembre de 2012 y el 1 de marzo de 2013 (73 días) en la Unidad Experimental Palo Pique perteneciente al Instituto Nacional de Investigación Agropecuaria (INIA) en la región este del Uruguay (S $33^{\circ} 15^{\prime} 38.77^{\prime \prime}$, W $54^{\circ}$ 29'45.34"). El manejo de los animales fue aprobado por la Comisión de Ética en el Uso de Animales de Experimentación de INIA. Se utilizaron 54 novillos de 1/1/2 año de edad cruza Hereford x Aberdeen Angus (peso inicial \pm DE: $304 \pm 44 \mathrm{~kg}$ ) distribuidos al azar en dos tratamientos con dos repeticiones (17 y 18 animales/ tratamiento, en repetición 1 y 2). Los tratamientos fueron pastoreo de 1) sudangras (Sorghum sudanense) cv
La Estanzuela Comiray, y 2) sorgo forrajero (Sorghum vulgare) nervadura marrón (BMR) cv AGT 21.

En cada grupo de animales el sistema de pastoreo fue rotativo en 4 parcelas de 0,75 ha cada una con una dotación instantánea de 23 y 24 animales/ha (repetición 1 y 2 , respectivamente). La fecha de siembra de ambos materiales fue el 6 de noviembre de 2012 a una densidad de $22 \mathrm{~kg} / \mathrm{ha}$ habiendo recibido el mismo manejo pre-siembra en cuanto a fertilización y aplicación de herbicidas.

A los 21 días post-siembra se cuantificó la cantidad de plantas por metro (m) lineal en las 4 parcelas en cada repetición mediante 10 muestreos al azar de $1 \mathrm{~m}$ cada uno por parcela. Se estimó el número de plantas por hectárea a una distancia entre hileras de $40 \mathrm{~cm}$. Luego de iniciado el pastoreo, en 2 de las 4 parcelas de cada repetición se determinó la altura del cultivo $(\mathrm{cm})$ y la disponibilidad (kg MS/ha) tanto al entrar (disponible) como al salir (remanente) los animales.

Para la estimación de la disponibilidad se utilizó un marco de $0.25 \mathrm{~m}^{2}(50 \times 50 \mathrm{~cm})$ empleando la técnica de doble muestreo ${ }^{14}$ utilizando 5 puntos de referencia y 15 puntos de estimación visual por parcela. Para estimar la cantidad de materia seca disponible dentro de cada marco de referencia se tomaron sub-muestras de 500 g las que fueron pesadas y secadas en estufa a $60^{\circ} \mathrm{C}$ durante 48 horas. Adicionalmente, se evaluó la composición de la biomasa mediante la separación de las fracciones hoja y tallo para determinar la proporción de éstos, así como la relación de ambos componentes en base seca.

Las muestras de hoja y tallo correspondientes de una parcela por ciclo de pastoreo por repetición se enviaron al Laboratorio de Nutrición Animal de INIA La Estanzuela para la determinación de proteína cruda (PC), fibra detergente ácido (FDA), fibra detergente neutra (FDN), cenizas $(\mathrm{C})$, y lignina (L). Se registró el peso vivo lleno $(\mathrm{kg})$ de los animales cada 21 días con el objetivo de estimar la ganancia de peso $(\mathrm{kg} / \mathrm{a} / \mathrm{d})$ por periodo de pastoreo y promedio en los $73 \mathrm{~d}$ mediante la regresión lineal del peso vivo en el tiempo.

Para el análisis estadístico se utilizó el paquete SAS versión 9.12 (SAS Institute, Cary, NC, USA). Las variables fueron sometidas a un análisis de varianza utilizando un diseño completamente al azar con dos repeticiones. Se utilizó un modelo que evaluó el efecto del tratamiento por periodo de pastoreo y/o promediando sobre todo el período experimental utilizando la sub-parcela (pasturas) y cada grupo de animales como unidad experimental. En todos los casos, las medias de los efectos fijos significativos $(\mathrm{p}<0,05)$ se separaron 
mediante el comando LSMEANS. Los resultados para cada variable se presentan como media \pm desvío estándar (DE).

\section{RESULTADOS}

A los 21 días post-siembra el sudangras registró una mayor cantidad de plantas por superficie $(p<0,05)$ comparado con el sorgo BMR (Tabla 1). A pesar de dicha diferencia, tanto la altura $(99 \pm 27 \mathrm{~cm})$ como la disponibilidad de forraje $(6409 \pm 2368 \mathrm{~kg} \mathrm{MS} / \mathrm{ha})$ al inicio del primer pastoreo ( $48 \mathrm{~d}$ post-siembra) fue igual para ambas variedades $(\mathrm{p}>0,05)$, si bien numéricamente la disponibilidad total del sorgo BMR fue $15 \%$ superior. Tampoco se registraron diferencias significativas $(p>0,05)$ en el aporte de hojas realizado a la materia seca total $(50 \%)$ ni en la tasa de crecimiento en el periodo siembra - primer pastoreo (131 $\pm 36 \mathrm{~kg} \mathrm{MS} /$ ha/día).

Durante el segundo y tercer pastoreo no se registraron diferencias significativas $(p>0,05)$ entre tratamientos en las variables altura, disponibilidad de forraje total y aporte de las fracciones hoja y tallo (Tabla 2). Al igual que en el primer pastoreo, la disponibilidad de forraje total del sorgo BMR fue numéricamente superior a la registrada en el sudangras (28\% y $21 \%$ superior en el segundo y tercer pastoreo, respectivamente). En ambas variedades, el aporte de la fracción hoja a la disponibilidad total disminuyó de un $50 \%$ en el primer pastoreo a un $20-25 \%$ durante el segundo y tercer pastoreo.

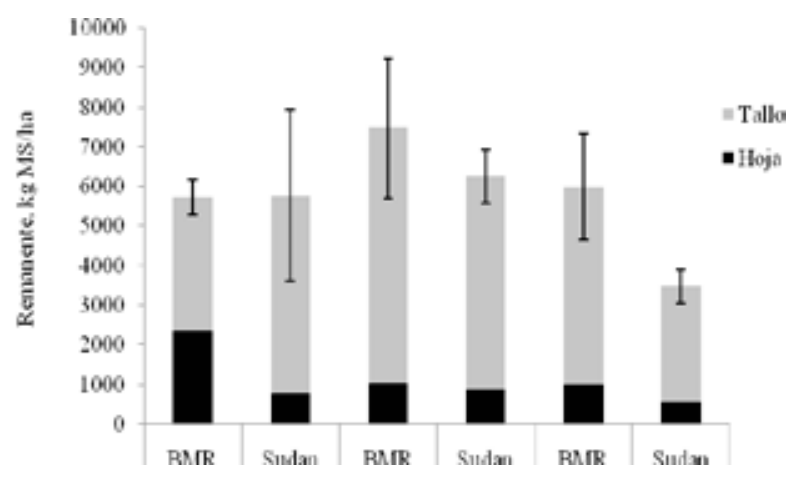

Figura 1. Forraje remanente al momento de retirar los animales luego de cada ciclo de pastoreo de sorgo BMR y sudangras. Líneas verticales en cada columna indican desviación estándar de la media de forraje total (hoja + tallo). bilidad total.
Tabla 1. Implantación y características al primer pastoreo de sudangras y sorgo BMR (media $\pm \mathrm{DE})$.

\begin{tabular}{lccc}
\hline & sudangras & sorgo BMR & $\mathrm{p}^{1}$ \\
\hline Implantación (21 d post-siembra) & & & \\
$\mathrm{N}^{\mathrm{o}}$ de plantas/m lineal & $25^{\mathrm{a}} \pm 3$ & $14^{\mathrm{b}} \pm 1$ & $<0,05$ \\
$\mathrm{~N}^{\mathrm{o}}$ de plantas/metro cuadrado & $63^{\mathrm{a}} \pm 7$ & $34^{\mathrm{b}} \pm 4$ & $<0,05$ \\
\hline Primer pastoreo (48 d post-siembra) & & & \\
altura, cm & $101 \pm 24$ & $98 \pm 34$ & $\mathrm{~ns}$ \\
forraje total, kg MS/ha & $5973 \pm 1947$ & $6845 \pm 2964$ & $\mathrm{~ns}$ \\
forraje hojas ${ }^{2}, \mathrm{~kg} \mathrm{MS} / \mathrm{ha}$ & $2972 \pm 1351(50)$ & $3374 \pm 1384(49)$ & $\mathrm{ns}$ \\
forraje tallos ${ }^{2}, \mathrm{~kg} \mathrm{MS} / \mathrm{ha}$ & $3001 \pm 880(50)$ & $3471 \pm 2241(51)$ & $\mathrm{ns}$ \\
tasa de crecimiento, kg MS/ha/d & $124 \pm 30$ & $137 \pm 45$ & $\mathrm{~ns}$ \\
\hline
\end{tabular}

${ }^{1}$ Letras diferentes en una misma fila indican diferencia significativa $(\mathrm{p}<0,05)$; ns: no significativa. ${ }^{2}$ Entre paréntesis: aporte porcentual de la fracción en la disponi-

Tabla 2. Altura y disponibilidad de forraje (media $\pm \mathrm{DE}$ ) al inicio del segundo y tercer pastoreo de sudangras y sorgo BMR.

\begin{tabular}{lccc}
\hline & sudangras & sorgo BMR & $\mathrm{p}^{1}$ \\
\hline Segundo pastoreo & & & \\
altura, cm & $95 \pm 9$ & $93 \pm 7$ & $\mathrm{~ns}$ \\
forraje total, kg MS/ha & $7451 \pm 1461$ & $9550 \pm 2932$ & $\mathrm{~ns}$ \\
forraje hojas ${ }^{2}, \mathrm{~kg} \mathrm{MS} / \mathrm{ha}$ & $1882 \pm 359(25)$ & $2493 \pm 1537(26)$ & $\mathrm{ns}$ \\
forraje tallos, kg MS/ha & $5569 \pm 1305(75)$ & $6607 \pm 1619(74)$ & $\mathrm{ns}$ \\
\hline Tercer pastoreo & & & \\
altura, cm & $84 \pm 3$ & $85 \pm 17$ & $\mathrm{~ns}$ \\
forraje total, kg MS/ha & $5712 \pm 644$ & $6935 \pm 2873$ & $\mathrm{~ns}$ \\
forraje hojas, $\mathrm{kg} \mathrm{MS} / \mathrm{ha}$ & $1389 \pm 142(24)$ & $1494 \pm 244(21)$ & $\mathrm{ns}$ \\
forraje tallos, $\mathrm{kg} \mathrm{MS} / \mathrm{ha}$ & $4323 \pm 624(76)$ & $5441 \pm 2653(79)$ & $\mathrm{ns}$ \\
\hline
\end{tabular}

${ }^{1}$ p $>0,05$ no significativo (ns); ${ }^{2}$ Entre paréntesis: aporte porcentual de la fracción en la disponibilidad total.

En cuanto al forraje remanente al momento de retirar los animales, no se registraron diferencias significativas $(p>0,05)$ entre tratamientos en altura y disponibilidad de forraje durante los tres ciclos de pastoreo (Figura 1). Numéricamente, si bien la altura del forraje remanente fue superior en sudangras $(82 \pm 13 \mathrm{~cm})$ comparado con el sorgo BMR $(75 \pm 8 \mathrm{~cm})$, la disponibilidad de forraje fue $21 \%$ mayor en el sorgo BMR comparado con el sudangras (6673 y $5502 \mathrm{~kg} \mathrm{MS} / \mathrm{ha}$, respectivamente). El aporte promedio de la fracción hoja a la materia seca total del forraje remanente fue de $24 \%$ (sorgo BMR) y 13\% (sudangras). Dicha diferencia fue generada en el forraje remanente del primer ciclo de pastoreo, en donde la disponibilidad de la fracción hoja fue significativamente mayor en el sorgo BMR comparado con el sudangras (2342 y $762 \mathrm{~kg} \mathrm{MS} / \mathrm{ha}$, respectivamente).

Se encontró una correlación alta y positiva $(\mathrm{p}<0,05)$ entre la altura del tapiz y el forraje disponible al inicio de los pastoreos tanto para sudangras $(\mathrm{r}=0,80)$ como para sorgo BMR $(r=0,80)$. El modelo de regresión lineal entre altura y disponibilidad también fue significativo $(\mathrm{p}<0,05)$, en donde cada $\mathrm{cm}$ adicional de altura correspondió a un incremento de 77 y $102 \mathrm{~kg}$ MS en sudangras $\left(\mathrm{R}^{2}=0,64\right)$, y sorgo BMR $\left(\mathrm{R}^{2}=0,60\right)$, respec- 
Tabla 3. Valor nutritivo (media \pm DE) de las fracciones hoja y tallo de sudangras y sorgo BMR en el forraje disponible (promedio de 3 ciclos de pastoreo).

\begin{tabular}{lcccccc}
\hline & \multicolumn{3}{c}{ fracción hoja } & \multicolumn{3}{c}{ fracción tallo } \\
\hline parámetro (\%) & sudangras & sorgo BMR & $\mathrm{p}^{2}$ & sudangras & sorgo BMR & $\mathrm{p}^{2}$ \\
MS & $27,4 \pm 3,5$ & $22,2 \pm 3,0$ & $<0,05$ & $19,4 \pm 5,4$ & $15,9 \pm 4,6$ & $\mathrm{~ns}$ \\
DIV & $64,5 \pm 2,3$ & $67,7 \pm 2,9$ & $<0,10$ & $61,5 \pm 5,0$ & $70,7 \pm 2,8$ & $<0,05$ \\
PC & $14,7 \pm 4,2$ & $15,1 \pm 3,2$ & $\mathrm{~ns}$ & $5,0 \pm 2,9$ & $6,8 \pm 3,3$ & $\mathrm{~ns}$ \\
FDA & $37,5 \pm 1,5$ & $38,8 \pm 2,5$ & $\mathrm{~ns}$ & $42,8 \pm 3,6$ & $39,4 \pm 2,3$ & $<0,10$ \\
FDN & $61,8 \pm 0,9$ & $63,7 \pm 2,3$ & $\mathrm{~ns}$ & $64,7 \pm 4,8$ & $60,2 \pm 3,6$ & $\mathrm{~ns}$ \\
cenizas & $9,2 \pm 1,0$ & $9,8 \pm 1,3$ & $\mathrm{~ns}$ & $8,1 \pm 2,0$ & $8,3 \pm 3,1$ & $\mathrm{~ns}$ \\
lignina & $11,6 \pm 1,0$ & $11,9 \pm 2,2$ & $\mathrm{~ns}$ & $9,3 \pm 1,0$ & $8,1 \pm 1,1$ & $<0,05$ \\
\hline
\end{tabular}

${ }^{1}$ MS: materia seca, DIV: digestibilidad in-vitro, PC: proteína cruda, FDA: fibra detergente ácida, FDN: fibra detergente neutro. ${ }^{2} \mathrm{p}<0,05$ diferencia significativa entre filas dentro de una fracción; $p>0,05$ sin diferencias significativas (ns); $p<0,10$ tendencia $(0,05 \leq \mathrm{p}<0,10)$.

Tabla 4. Desempeño productivo (media $\pm \mathrm{DE}$ ) de novillos pastoreando sudangras o sorgo BMR.

\begin{tabular}{lccc}
\hline & \multicolumn{2}{c}{ tratamiento } \\
\hline & sudangras & sorgo BMR & $\mathrm{p}^{1}$ \\
peso vivo inicial, $\mathrm{kg}$ & $320 \pm 28$ & $320 \pm 29$ & $\mathrm{~ns}$ \\
peso vivo final, kg & $378 \pm 27$ & $388 \pm 28$ & $\mathrm{~ns}$ \\
ganancia de peso, kg/a/d & & & \\
0-21 d & $0,796^{\mathrm{a}} \pm 0,375$ & $1,082^{\mathrm{b}} \pm 0,418$ & 0,009 \\
21-42 d & $1,034 \pm 0,542$ & $1,091 \pm 0,453$ & $\mathrm{~ns}$ \\
42-73 d & $0,669 \pm 0,428$ & $0,708 \pm 0,304$ & $\mathrm{~ns}$ \\
$0-73 \mathrm{~d}$ & $0,823^{\mathrm{a}} \pm 0,155$ & $0,945^{\mathrm{b}} \pm 0,184$ & 0,004 \\
\hline
\end{tabular}

${ }^{1}$ Letras diferentes en una misma fila diferencia significativa $(\mathrm{p}<0,05)$; ns: no significativo.

tivamente. Las mismas tendencias fueron registradas en el forraje remanente, en donde por cada un $\mathrm{cm}$ de incremento en altura la disponibilidad se incrementó 67 (sudangras, $\mathrm{R}^{2}=0,60$ ) y $79 \mathrm{~kg}$ MS (sorgo BMR, $\mathrm{R}^{2}=0,49$ ).

Comparando el valor nutritivo de la fracción hoja, el sorgo BMR presentó un menor porcentaje de materia seca $(p<0,05)$ y tendió a registrar una mayor digestibilidad que el sudangras $(p<0,10)$, no encontrándose diferencias significativas $(p>0,05)$ en el resto de los parámetros (Tabla 3). En la fracción tallo el sorgo BMR registró un incremento de $15 \%$ en la digestibilidad y un descenso de $13 \%$ en el contenido de lignina comparado con el sudangras $(p<0,05)$ y una tendencia a menor contenido de fibra detergente ácida $(p<0,10)$. Como característica diferencial entre ambas variedades, en el sorgo BMR la hoja registró menor digestibilidad y mayor fibra detergente neutro que el tallo, mientras que en el sudangras la fracción hoja presentó mayor digestibilidad y menor fibra detergente neutro que la fracción tallo.

La digestibilidad de la hoja y tallo del sorgo BMR tendió a mantenerse estable e incluso a incrementarse levemente (1-4\%) al pasar del primer al tercer ciclo de pastoreo, a diferencia de las fracciones de sudangras que redujeron su digestibilidad entre un 5\% (hoja) y
$15 \%$ (tallo). La lignina en la fracción tallo del sorgo BMR disminuyó de 9,1 a 7,8\% del primer al tercer pastoreo respectivamente, mientras que se mantuvo constante en el tallo de sudangras $(9,3 \%)$. También en el tallo, la proteína cruda disminuyó un $64 \%$ (9,8 y 3,5\% respectivamente) al pasar del primer al tercer pastoreo, promediando sobre ambas variedades.

$\mathrm{Si}$ bien no existieron diferencias significativas $(p>0,05)$ en el peso vivo de los animales al finalizar el experimento, los animales del tratamiento sorgo BMR fueron $10 \mathrm{~kg}$ más pesados que aquellos del sudangras (Tabla 4). Esto fue debido a una mayor $(p<0,05)$ ganancia diaria de peso de los animales en el sorgo BMR comparado con el sudangras en los 73 días de pastoreo $(0,945$ y $0,823 \mathrm{~kg} / \mathrm{a} / \mathrm{d}$ respectivamente). Si se analiza la ganancia de peso por periodo, la diferencia significativa $(\mathrm{p}<0,05)$ se registró en los primeros 21 días $(1,082$ y $0,796 \mathrm{~kg} / \mathrm{a} /$ día, sorgo BMR y sudangras respectivamente) durante el primer pastoreo. Luego, durante el segundo y tercer pastoreo, la superioridad en ganancia de peso de los animales en el sorgo BMR se mantuvo relativamente constante, cercana al $6 \%(p>0,05)$. La producción de peso vivo por superficie estimada fue 326 y $375 \mathrm{~kg} / \mathrm{ha}$ para sudangras y sorgo BMR respectivamente.

\section{DISCUSIÓN}

A pesar de haber utilizado la misma densidad de siembra (22 kg/ha), el número de plantas fue mayor en el sudangras que en el sorgo BMR. Esto fue debido a un menor peso absoluto y tamaño de la semilla de sudangras. Sin embargo, en el primer pastoreo no hubo diferencias significativas en la disponibilidad de forraje y altura del tapiz entre ambos materiales. Si bien se ha reportado que los materiales que incluyen el gen BMR pueden producir menos materia seca total que aquellos 
sin la mutación ${ }^{4,7}$, en el presente experimento la disponibilidad de forraje fue entre un 15 y $28 \%$ superior en el sorgo BMR comparado con el sudangras, considerando los tres periodos de pastoreo.

Aunque no se evaluó la producción total de forraje, la disponibilidad de materia seca al inicio de cada ciclo de pastoreo puede ser un buen indicador de la producción total bajo una misma dotación y manejo del pastoreo. A pesar de que ambos materiales presentaron similar altura del tapiz, por cada $\mathrm{cm}$ de incremento el sorgo BMR presentó una mayor concentración de materia seca (102 y $77 \mathrm{~kg} \mathrm{MS} / \mathrm{cm}$ respectivamente) lo que explicó la mayor disponibilidad de forraje. La misma tendencia se registró en el forraje remanente en donde el sorgo BMR registró un valor promedio de disponibilidad de forraje $21 \%$ superior que el sudangras con una mayor concentración de materia seca por cada $\mathrm{cm}$ de altura.

El descenso del componente hojas al avanzar el ciclo de producción en ambos materiales se atribuyó al manejo poco flexible del pastoreo con dotación y tiempo de ocupación fijo, y a la altura del forraje remanente ${ }^{6,8,9}$. Debido al mayor valor nutritivo de la hoja comparado con el tallo, era de esperar un descenso en el desempeño productivo de los animales al avanzar el ciclo de producción, con una mayor predominancia de tallos ${ }^{28}$. Como consecuencia, la ganancia de peso disminuyó un $27 \%$ al pasar del primer al tercer ciclo de pastoreo, promediando sobre ambos materiales.

Desde el punto de vista nutricional el principal parámetro responsable del descenso en el desempeño productivo de los animales fue la proteína cruda, la cual disminuyó al avanzar el ciclo de producción, coincidentemente con lo reportado por otros autores ${ }^{5,10}$. Promediando sobre ambos materiales evaluados, al iniciar el tercer ciclo de pastoreo el contenido de proteína en la hoja $(12,9 \%)$ y tallo $(3,5 \%)$ fue significativamente menor a los valores registrados en el primer pastoreo ( $19,3 \%$ y $9,8 \%$ respectivamente). Debido a que la fracción tallo representó más del $70 \%$ al finalizar el ciclo de producción, la oferta total de proteína se consideró limitante para un adecuado crecimiento de los animales.

Cuando el forraje fresco tiene un contenido proteico inferior al $11 \%$ puede haber deficiencias de nitrógeno a nivel ruminal para la síntesis de proteína microbiana ${ }^{15}$, lo que se vio reflejado en la disminución de la ganancia de peso en el último pastoreo. El sorgo BMR presentó como ventaja mayor digestibilidad y menor contenido de lignina en el tallo, lo que mejoró la ganancia de peso de los novillos en dicho tratamiento. El carácter indigestible de la lignina explicó el incremento de digestibilidad al disminuir la primera ${ }^{22}$, característica que ha sido reportada por varios autores en el sorgo BMR $^{1,12,13,24}$. Además de la mayor digestibilidad, el tallo del sorgo BMR registró una tendencia a menores valores de fibra detergente, tanto neutra (FDN) como ácida (FDA), con implicancias en el consumo de materia seca y de energía.
De acuerdo a los valores de FDN, el consumo teórico potencial de materia seca del tallo sería de 1,8\% (sudangras) y 2,0\% (sorgo BMR) del peso vivo según ecuación publicada para estimar el consumo diario de alimentos fibrosos ${ }^{27}$. Adicionalmente, varios trabajos reportaron que la digestibilidad de la fracción FDN en sorgos con el gen BMR es mayor comparado con materiales similares que no poseen dicho gen ${ }^{2,13,24}$ resultando en un incremento del consumo de energía, incluso a igual valor de FDN ${ }^{23}$. Relacionado al consumo de energía, el menor valor de FDA en el tallo del sorgo BMR es un indicador de la mayor digestibilidad comparado con el tallo del sudangras.

De esta manera, un mayor consumo de la fracción tallo del sorgo BMR con una digestibilidad y concentración energética mayor que el sudangras estaría explicando en parte la superioridad de la tasa de ganancia observada en los animales pastoreando el sorgo BMR. El mayor valor nutritivo del sorgo BMR comparado con otras variedades de sorgo y sudangras fue corroborado por experimentos de comportamiento de pastoreo, en donde animales expuestos a elegir distintos materiales genéticos al mismo tiempo (prueba de preferencia), siempre pastorearon como primera opción y utilizaron una mayor proporción del forraje de aquellos materiales que incluían el gen BMR ${ }^{21}$.

El mejor desempeño productivo de los novillos pastoreando el sorgo con el gen BMR en el presente experimento coincide tanto en la tendencia como en la magnitud, con trabajos en donde la ganancia de peso de animales con acceso a materiales BMR fue entre un 12 y $17 \%$ superior que aquellos animales pastoreando los mismos materiales pero sin el gen BMR ${ }^{3,20}$. En otra serie de experimentos de 2 años de duración, novillos manejados a una dotación conservadora (4 animales/ ha) sobre sorgo BMR registraron un desempeño productivo superior que novillos manejados sobre sorgo foto-sensitivo pero sin el gen BMR $(1,180$ y $0,900 \mathrm{~kg} /$ a/d respectivamente) ${ }^{19}$. Si bien en el presente trabajo se evaluaron dos materiales forrajeros distintos (sorgo forrajero y sudangras) que genotípicamente difieren más allá del gen BMR, la inclusión de esta característica presentó una ventaja productiva en términos de producción de carne que compensó el incremento del costo de la semilla, comparado con materiales tradicionales como el sudangras con menor potencial de producción.

Como conclusión, en un escenario de intensificación de la producción ganadera como estrategia de incremento de competitividad de la empresa, el uso de sorgos forrajeros con el gen BMR permite mejorar el desempeño productivo de novillos en pastoreo durante el verano.

\section{REFERENCIAS}

1. Akin D, Hanna W, Sook M, Himmelsbach D, Barton F, Windham WR. 1986. Normal 12 and brown midrib 12 sorghum; chemical variations and digestibility. Agron $J$ 78: 832-837. 
2. Aydin G, Grant RJ, O'Rear J. 1999. Brown midrib sorghum in diets for lactating dairy cows. $J$ Dairy Sci 82 : 2127-2135.

3. Banta JB, McCollum FT, Greene LW. 2005. The effects of grazing a brown midrib vs a conventional sorghum $\mathrm{x}$ sudan hybrid on animal performance and gain/ha. J Anim Sci 79 (Suppl.1): 458.

4. Bean BW. 2007. Producing quality forage sorghum silage. Proceedings Southern Corn and Rice Minimum Tillage Conference, Houston, Texas, USA. On line: http://amarillo.tamu.edu/files/2010/11/ProducingQualityForageSorghumSilage.pdf

5. Beck PA, Hutchison S, Gunter SA, Losi TC, Stewart CB, Capps PK, Phillips JM. 2007. Chemical composition and in situ dry matter and fiber disappearance of sorghum $\times$ Sudangrass hybrids. J Anim Sci 85: 545-555.

6. Berlangieri MS. 2008. Efecto del manejo y el material genético en la productividad de sorgo forrajero bajo pastoreo. Tesis de Grado, Facultad de Agronomía, Universidad de la República. Montevideo, Uruguay, 150 p.

7. Casler MD, Pederson F, Undersander DJ. 2003. Forage yield and economic losses associated with the brown-midrib trait in sudangrass. Crop Sci 43: 782-789.

8. Edwards NC, Henry JR, Fribourg A, Montgomery M. 1971. Cutting management effects on growth rate and dry matter digestibility of sorghum sudangrass cultivar Sudax SX 11. Agron J 63: 267-271.

9. Espinoza M, Argenti P, Gil J, Perdomo E, León L. 1992. Rendimiento y calidad nutritiva de cuatro híbridos y una variedad de sorgo forrajero (Sorghum bicolor pers.) bajo riego complementario. Zootec Trop 10: 171-188.

10. Fernández Mayer AE, Stuart RJ, Chongo B, Martin PC. 2011. Ceba pastoril con sorgos nervadura marrón o BMR (Brown Middle Rib) como forraje fresco. Rev Cuba Cien Agri 45: 251-256.

11. Frenchick GE, Jhonson DG, Murphy JM, Otterby DE. 1976. Brown midrib corn silage in dairy cattle rations. $J$ Dairy Sci 59: 2126-2129.

12. Fritz JO, Cantrell RP, Lechtenberg VL, Axtel JD, Hertel JM. 1981. Brown midrib mutants in Sudangrass and grain Sorghum. Crop Sci 21: 706-709.

13. Gerhardt RL, Fritz JO, Jaster EH, Moore KJ. 1994. Digestion kinetics and composition of normal and brown midrib sorghum morphological components. Crop Sci 34: 1353-1361.

14. Haydock KP, Shaw NH. 1975. The comparative yield method for estimating the dry matter yield of pasture. Australian J Exper Agric Animal Husb 15: 663-670.

15. Hoover WH, Stokes SR. 1991. Balancing carbohydrates and proteins for optimum rumen microbial yield. J Dairy Sci 74: 3630-3644.
16. Jung HG, Fahey GC. 1983. Nutritional implications of phenolic monomers and lignin: a review. J Anim Sci 57: 206-219.

17. Jung HG, Fahey GC. 1983. Interactions among phenolic monomers and in vitro fermentations. J Dairy Sci 66: 1255-1263.

18. Keith EA, Colenbrander VF, Lechtenberg VL, Bauman LF. 1979. Nutritional value of brown midrib corn silage for lactating dairy cows. J Dairy Sci 62: 788-792.

19. McCuistion KC, McCollum FT, Greene LW, Bean B, Van Meter R, Vasconcelos J, Silva J. 2005. Performance of steers grazing photoperiod-sensitive and brown midrib varieties of sorghum-sudangrass. Forage Sorghum Field Day, Texas Agricultural Research and Extension Center, Amarillo (USA).

20. McCuistion KC, McCollum FT, Greene LW, MacDonald J, Bean B. 2011. Performance of stocker cattle grazing 2 sorghum-sudangrass hybrids under various stocking rates. Prof Anim Sci 27: 92-100.

21. Miller FR, Stroup JA. 2004. Growth and management of sorghums for forage production. Proceedings of the National Alfalfa Symposium, 13-15 December, 2004, San Diego, $C A$, UC Coop. Ext., Univ. of California, Davis, CA (USA).

22. Moore KJ, Jung HG. 2001. Lignin and fiber digestion. $J$ Range Manage 54: 420-430.

23. Oba M, Allen MS. 1999. Evaluation of the importance of the digestibility of neutral detergent fiber from forage: effects on dry matter intake and milk yield of dairy cows. $J$ Dairy Sci 82: 589-596.

24. Oliver L, Pedersen JF, Grant RJ, Klopfenstein TJ. 2005. Comparative effects of the sorghum bmr- 6 and bmr12 genes. Crop Sci 45: 2234-2239.

25. Porter KS, Axtell JD, Lechtenberg VL, Colenbrander VF. 1978. Phenotype, fiber composition, and in vitro dry matter disappearance of chemically induced brown midrib mutants of sorghum. Crop Sci 18: 205-208

26. Stallings CC, Donaldson BM, Thomas JW, Rosman EC. 1982. In vitro evaluation of brown midrib corn silage by sheep and lactating dairy cows. J Dairy Sci 65: 19451949.

27. Undersander D, Moore JE. 2002. Relative forage quality (RFQ) indexing legumes and grasses for forage quality. University of Wisconsin Extension. Disponible on line: http://www.uwex.edu/ces/forage/pubs/rfq.htm.

28. Vasconcelos JT, Greene LW, McCollum FT, Bean BW, Van Meter R. 2003. Performance of crossbred steers grazing photoperiod sensitive and non photoperiod sensitive Sorghum Sudangrass hybrids. J Anim Sci 81(suppl. 2): 103. 